\title{
Effet de la fréquence de récolte et des milieux de dilution sur la qualité du sperme de taureau de race Borgou
}

\author{
A.B. GBANGBOCHE ${ }^{1}$, T. I. ALKOIRET ${ }^{2 *}$, C.A.A.M. CHRYSOSTOME ${ }^{1}$, \\ J. DOSSOU-BODJRENOU ${ }^{1}$, E. AISSI ${ }^{1}$, A. ADJOVI ${ }^{1}$, M. ADAMOU-N'DIAYE ${ }^{1}$ et \\ J.-L. BISTER ${ }^{3}$ \\ ${ }^{1}$ Laboratoire de Biotechnologie et d'Amélioration Animales - Faculté des Sciences Agronomiques- Université \\ d'Abomey Calavi-01Bp 526 Cotonou-Bénin. \\ ${ }^{2}$ Laboratoire de Production et Nutrition Animales - Département des Productions Animales - Faculté des \\ Sciences Agronomiques - Université de Parakou, BP 123 Parakou, Bénin. \\ ${ }^{3}$ Laboratoire de Physiologie animale - Facultés Universitaires Notre-Dame de la Paix. B-5000 Namur- \\ Belgique. \\ *Auteur correspondant, E-mail : alkoiretib@yahoo.fr
}

\section{RESUME}

La présente étude a pour but d'évaluer la production et la qualité de sperme de 17 taureaux Borgou. L'expérimentation a été réalisée sur la base des séances de récolte tous les 4 jours, pendant 36 jours au cours desquels l'efficacité in vitro de six milieux de dilution sur la motilité et la vitalité des spermatozoïdes (spz) a été testée pendant 28 jours. Les résultats ont montré que la fréquence de récolte expérimentée n'affecte pas la qualité des éjaculats qu'on pourrait utiliser pour l'insémination artificielle à l'état frais $\left(+5^{\circ} \mathrm{C}\right)$. L'effet des différents dilueurs sur la motilité et la viabilité des spermatozoïdes, a prouvé que ces spermatozoïdes supportaient moins bien le milieu à base de lait de coco que ceux à base de jaune d'œuf et de lait de vache. Cependant, tous les milieux de dilution testés peuvent être utilisés pour conserver les semences $+5{ }^{\circ} \mathrm{C}$ durant 48 heures. La durée de conservation peut être étendue à 72 heures pour le lait de vache et le jaune d'œuf. (c) 2011 International Formulae Group. All rights reserved.

Mots clés: Sperme, insémination artificielle, dilueurs, éjaculats, bovin, Benin.

\section{INTRODUCTION}

Parmi les problèmes qui intéressent au plus haut point l'élevage au Bénin, se trouvent ceux relatifs à l'utilisation de l'insémination artificielle, comme outil de diffusion de progrès génétique. Sa complexité laisse entrevoir une multiplicité de causes susceptibles d'entraîner l'infécondité et des pertes économiques. Qu'un seul maillon de la technologie de sperme (récolte, dilution, conservation et insémination) soit défectueux et le résultat final de l'insémination artificielle, c'est à dire la fécondation, peut être compromise (Jondet 1980). C'est à juste titre que les milieux suivants sont testés pour la conservation de semence des ruminants: les milieux lactés (Martin, 1966 ; Leboeuf et al., 1998) ; les milieux à base de jaune d'œuf (Iritani et Nishikawa., 1961, 1963 ; Jondet, 1980 ; Moussa et al., 2002); les milieux glucosés (Palmer, 1984); les milieux à base de lait de 
coco (Norman et al., 1962). L'importance de la fréquence de récolte est explorée depuis très longtemps, pour valoriser la production de semence dans les centres d'insémination artificielle (Jondet, 1980). Le choix de la température pour la conservation des semences bovines, est mis en exergue depuis l'époque qui a précédé l'ère de la congélation. Ainsi, parmi les températures mises à l'épreuve comme $+20{ }^{\circ} \mathrm{C},+15{ }^{\circ} \mathrm{C}$, $+10{ }^{\circ} \mathrm{C}$ et $+5{ }^{\circ} \mathrm{C}$ pour la conservation de la semence, celle de $+5{ }^{\circ} \mathrm{C}$ s'est révélée la plus efficace et a montré après un réchauffement à $+38{ }^{\circ} \mathrm{C}$, le meilleur taux de survie des spermatozoïdes (Mcfee et Swanson, 1960). Concernant la race bovine Borgou, des études ont permis de caractériser les semences et leur potentiel d'utilisation en insémination artificielle (Adamou N'Diaye et al., 2000 et 2003), sans avoir à ce jour, recherché les milieux de dilution peu coûteux, disponibles, faciles à préparer et ceux qui se prêtent mieux aux conditions d'asepsie. C'est dans ce contexte que se situe la présente étude, dont le but est de tester in vitro, l'efficacité du citrate/fructose/jaune d'œuf, du lait de vache, du lait UHT commercial, du lait de coco, du glucose $5 \%$ et du glucose $10 \%, 6$ milieux de dilution, sur la mobilité et le pourcentage de spermatozoïdes vivants de taureaux de race Borgou.

\section{MATERIEL ET METHODES}

\section{Les animaux donneurs de semences}

Dix sept (17) taureaux reproducteurs de race Borgou préalablement entrainés à la récolte de sperme et régulièrement collectés au Centre d'Elevage et d'Insémination Artificielle de la Faculté des Sciences Agronomiques de l'Université d'Abomey Calavi sont utilisés. Les animaux sont âgés de $48 \pm 7$ mois. Toutefois, $6 \%$ des animaux avaient entre 30 et 45 mois mais $94 \%$ d'entre eux avaient entre 45 et 60 mois. Le poids vif corporel moyen \pm écart type des animaux était de $235 \pm 18 \mathrm{~kg}$. En effet, $82 \%$ des taureaux pesaient entre 200 et $250 \mathrm{~kg}$ et $18 \%$ des taureaux pesaient entre 250 et $270 \mathrm{~kg}$. Les animaux sont alimentés essentiellement sur pâturages naturels, à base de graminées locales. Les bovins expérimentaux n'ont reçu aucune complémentation alimentaire autre que les minéraux apportés par les pierres à lécher composées du phosphate bi calcique, du chlorure de sodium et de potassium, du carbonate de zinc, du sulfate de cuivre et du soufre. En dehors du temps passé au pâturage qui a été de 4 h 30 dans la matinée et 3 h 30 l'après midi, les animaux sont maintenus aux piquets dans un parc collectif pourvu d'un espace couvert.

\section{Récolte et examen des éjaculats : étude de l'effet de la fréquence de collecte}

Les éjaculats sont récoltés à raison de deux collectes par taureau, tous les matins entre $7 \mathrm{~h}$ et 10 heures avant la mise au pâturage, en présence de la femelle «boute en train », à l'aide d'un vagin artificiel. La température ambiante a varié de 24 à $30{ }^{\circ} \mathrm{C}$. Le temps de préparation des animaux a duré environ 10 minutes et comprenait une ou deux fausses montes pour leur mise en condition physique. Chaque éjaculat est aussitôt examiné après la récolte, pour enregistrer le volume, la couleur et la consistance. Pour évaluer l'effet de la fréquence de collecte sur les paramètres spermatiques, un intervalle de 4 jours correspondant à 1 séance/4 jours, est observé entre deux séances de récoltes successives. Ainsi, en définitive toute la collecte correspondait à 10 séances/36 jours ou à 2 récoltes/séance/taureau ou à 34 récoltes/séance, soit au total 340 éjaculats. Les éjaculats ayant présenté les caractéristiques suivantes sont conservés pour la dilution : volume $\geq 2 \mathrm{ml}$; concentration $\geq 0,4 \times 10^{9}$ spermatozoïdes $/ \mathrm{ml}$; pourcentage de spermatozoïdes vivants $\geq 60 \%$. Pour l'analyse des éjaculats, une goutte de sperme est examinée au microscope avec un grossissement 40 à 100 et sur platine une chauffante à $+37 / 38{ }^{\circ} \mathrm{C}$ pour apprécier la motilité notée subjectivement de 0 à 5 . La concentration est déterminée à l'aide d'une cellule hématimétrique par comptage des spermatozoïdes dans une goutte de sperme dilué à $1 \%$ avec une solution de chlorure de sodium à $3 \%$. Le pourcentage de 
spermatozoïdes vivants est évalué sous microscope après une coloration à l'éosinenigrosine.

\section{Mise au point des dilueurs et de la dilution et examen des semences : étude de l'effet du dilueur}

Six milieux de dilution préalablement chauffés sont refroidis de $+67{ }^{\circ} \mathrm{C}$ à $+5{ }^{\circ} \mathrm{C}$ correspondant à la température de conservation. La composition des milieux de dilution est décrite dans le tableau 1. Pour l'obtention du lait de coco, l'amande de la noix de coco est lavée à l'eau bidistillée et transformée en farine par mouture. Ainsi, 100 $\mathrm{g}$ de farine d'amande de coco sont macérés dans $100 \mathrm{ml}$ d'eau bidistillée soit $1 \mathrm{~g} / \mathrm{ml}$, puis filtrés. Le lait de vache Borgou est chauffé au bain-marie à $+92{ }^{\circ} \mathrm{C}-+95{ }^{\circ} \mathrm{C}$ pendant 10 minutes, puis centrifugé à 3.500 tours/minutes pendant 10 à 15 minutes pour en extraire la matière grasse. 60 des 68 éjaculats de la première et de la deuxième journée de récolte sont soumis à la dilution à raison de 10 éjaculats/par type de dilueurs. Ainsi, les 336 paillettes des doses de semences produites sont conservées à $+5{ }^{\circ} \mathrm{C}$, à raison de 56 paillettes par type de dilueur. Le volume de dilueur (concentration du sperme $\mathrm{x}$ volume $\mathrm{du}$ sperme) et le taux de dilution (concentration finale de la semence $x$ concentration initiale du sperme) sont calculés de sorte que la concentration finale de chaque semence soit de $80 \times 10^{6}$ spermatozoïdes $/ \mathrm{ml}$. La dilution est faite en deux phases successives. Une première dilution ou dilution partielle avec une première moitié du dilueur à $160 \times 10^{6}$ spermatozoïdes/ml suivi d'un refroidissement progressif de la semence pendant 2 heures de $+32{ }^{\circ} \mathrm{C}$ à $+5^{\circ} \mathrm{C}$ et ce sous vitrine réfrigérante. La dilution finale pour atteindre $80 \times 10^{6}$ spermatozoïdes/ml est réalisée par l'adjonction de la deuxième moitié du dilueur en 5 étapes de 10 minutes d'intervalle pour éviter les chocs osmotiques. Pendant 28 jours (J0-J28), deux paillettes/dilueur sont tirées au hasard et analysées afin de déterminer l'évolution journalière de la motilité et du pourcentage de spermatozoïdes vivants.

\section{Analyse statistique}

La procédure du modèle linéaire générale (proc GLM) du logiciel SAS ${ }^{\circledR} 9.2$ (2008) est appliquée au volume, à la motilité, à la concentration et au pourcentage de spermatozoïdes vivants. Les deux catégories de facteurs fixes de variation suivantes sont considérées : la fréquence de récolte (F1 à $\mathrm{F} 10)$; le nombre de récolte par séance (R1 et R2) pour les paramètres spermatiques ; le type de dilueurs (D1 à D6) pour la motilité et le pourcentage de spermatozoïdes vivants. Les valeurs moyennes sont comparées à l'aide du test t de Student et l'effet des facteurs fixes de variation est estimé au seuil de 5\%.

\section{RESULTATS}

Les paramètres spermatiques tels que le volume, la motilité, le pourcentage de spermatozoïdes vivants et la concentration sont restés statistiquement inchangés ( $p>0,05)$ avec les jours de récoltes et le nombre de récolte par séance (Tableau 2). Quant à l'évolution de la longévité (taux de survie) et de la motilité des spermatozoïdes dans les différents milieux de dilution, à J3, les semences ont présenté en moyenne 3,9 \pm 0,2 de motilité et $73,6 \pm 11,0 \%$ de spermatozoïdes vivants (Figures 1 et 2). Par ailleurs, le profil des Figures 1 et 2, était similaire dans les milieux à base de lait de vache et de jaune d'œuf. Jusqu'à J10, les pertes journalières de spermatozoïdes dans le milieu à base de lait de commerce étaient identiques à celles des dilueurs à base de lait de vache et de jaune d'œuf. Les pertes de spermatozoïdes et de leur motilité ont été statistiquement identiques $(\mathrm{p}>0,05)$ dans les milieux à base de glucose $5 \%$ et $10 \%$. En définitive, les spermatozoïdes ont supporté moins bien le milieu à base de lait de coco que ceux à base de jaune d'œuf et de lait de vache (Figures 1 et $2 ; \mathrm{p}<0,05$ ), puisque les pertes ont atteint $100 \%$ à partir de $\mathrm{J} 8$ dans le lait de coco, contre 5\% à J10 dans les dilueurs à base de glucose, contre $10 \%$ à $\mathrm{J} 12$ dans le lait écrémé (Figures 1 et 2). 


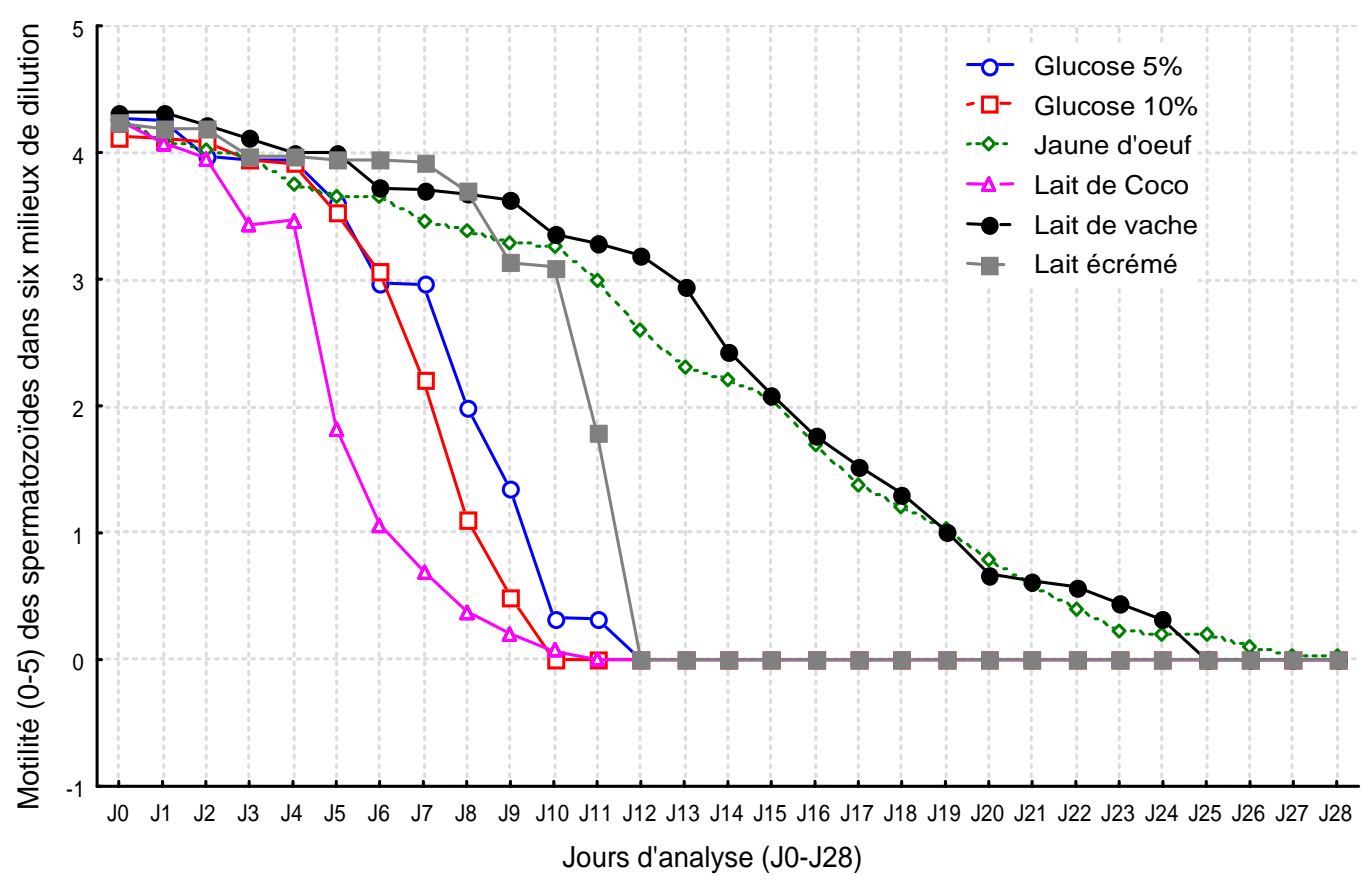

Figure 1 : Evolution de la motilité des spermatozoïdes (échelle de 0 à 5) dans six milieux de dilution, conservation à $+5^{\circ} \mathrm{C}$ pendant 28 jours.

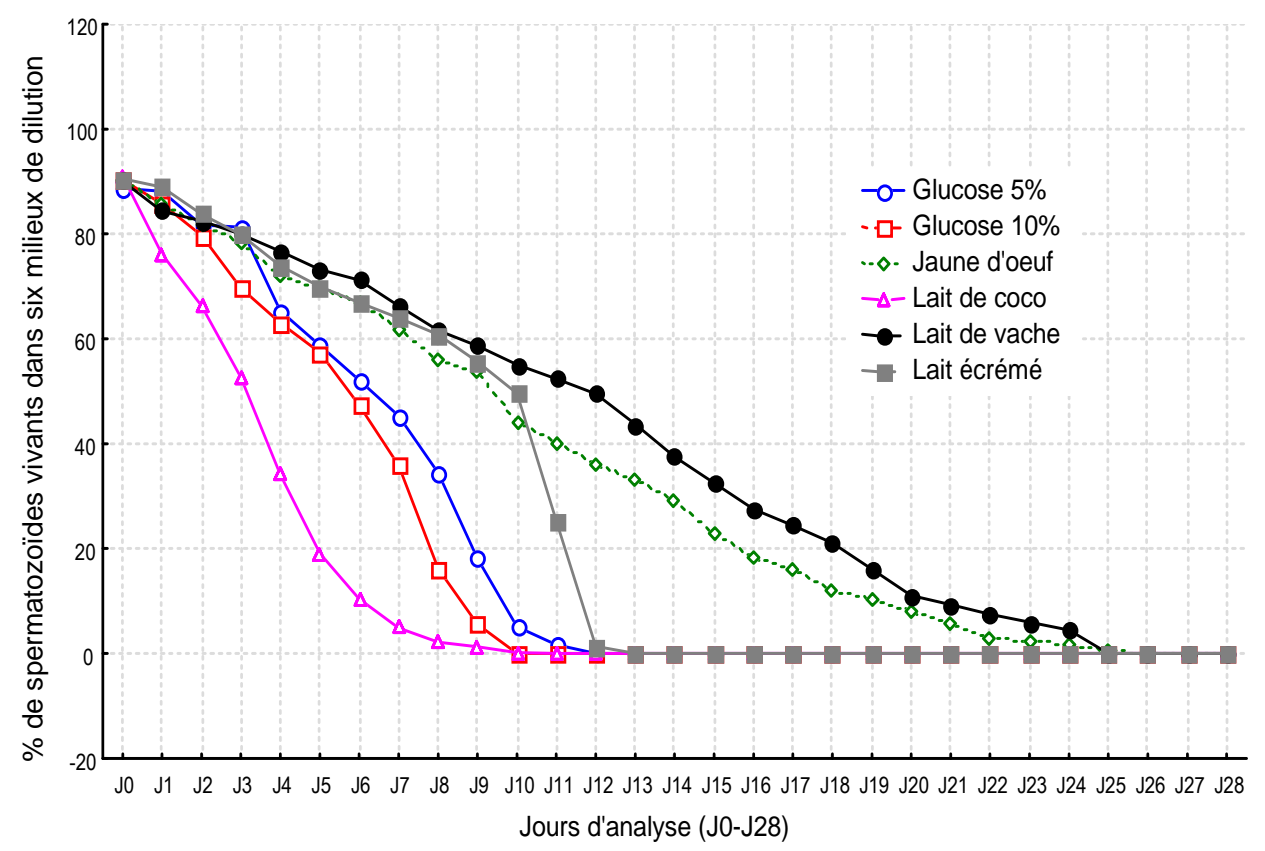

Figure 2 : Evolution de la viabilité de spermatozoïdes (\% de spermatozoïdes vivants) dans six milieux de dilution, conservation à $+5{ }^{\circ} \mathrm{C}$ pendant 28 jours. 
A. B. GBANGBOCHE et al. / Int. J. Biol. Chem. Sci. 5(5): 1871-1882, 2011

Tableau 1 : Composition de six milieux de dilution expérimentés sur des éjaculats de taureau de race Borgou au Bénin.

\section{Composants}

Dilueurs

\begin{tabular}{|c|c|c|c|c|c|c|}
\hline & $\begin{array}{l}\text { Lait de } \\
\text { vache }\end{array}$ & $\begin{array}{l}\text { Lait UHT de } \\
\text { commerce }\end{array}$ & Lait de coco & $\begin{array}{l}\text { citrate/fructose/ } \\
\text { jaune d'oeuf }\end{array}$ & Glucose 5\% & Glucose $10 \%$ \\
\hline Citrate de sodium $(\mathrm{g})$ & & & 22 & 25,3 & & \\
\hline Jaune d'œuf (ml) & 25 & 25 & 25 & 250 & 150 & 150 \\
\hline Pénicilline G (UI) & 1.000 .000 & 1.000 .000 & 2.000 .000 & 1.000 .000 & 1.000 .000 & 1.000 .000 \\
\hline $\begin{array}{l}\text { Streptomycine sulfate } \\
\text { (UI) }\end{array}$ & 2.000 .000 & 2.000 .000 & 3.000 .000 & 2.000 .000 & 2.000 .000 & 2.000 .000 \\
\hline Eau bi distillée $(\mathrm{ml})$ & & & 100 & 750 & 600 & 600 \\
\hline Fructose $(\mathrm{g})$ & & & & 20 & & \\
\hline Glycérol (ml) & 10 & 10 & & 40 & 10 & 20 \\
\hline Lait & 70 & 70 & & & & \\
\hline Lait de coco $(\mathrm{ml})$ & & & 150 & & & \\
\hline Glucose $(\mathrm{ml})$ & & & & & 100 & 200 \\
\hline Mycostatine (UI) & & & 1500 & & & \\
\hline $\mathrm{pH}$ & 7,0 & 7,2 & 7,1 & 6,75 & 6,50 & 6,80 \\
\hline & \multicolumn{2}{|c|}{$\begin{array}{c}\text { Modifié à partir de } \\
\text { Almquiste et al., } 1954 .\end{array}$} & $\begin{array}{c}\text { Modifié à } \\
\text { partir de } \\
\text { Norman et al., } \\
1962 .\end{array}$ & Jondet, 1980 & \multicolumn{2}{|c|}{ Modifié à partir de Dunn et al., 1953} \\
\hline
\end{tabular}


Tableau 2 : Caractéristiques spermatiques des éjaculats (moyenne \pm écart type) de taureaux de race Borgou en fonction de la fréquence du nombre de récolte par séance.

\begin{tabular}{|c|c|c|c|c|c|}
\hline \multirow{2}{*}{$\begin{array}{l}\text { Fréquence de } \\
\text { récolte }(n=10)\end{array}$} & \multirow{2}{*}{$\begin{array}{c}\text { Nombre de } \\
\text { récolte } \\
(\mathbf{n}=\mathbf{2 0})\end{array}$} & \multicolumn{4}{|c|}{ Paramètres spermatiques (moyenne \pm écart type) } \\
\hline & & $\begin{array}{l}\text { Volume } \\
(\mathrm{ml})\end{array}$ & $\begin{array}{c}\text { Motilité } \\
(05)\end{array}$ & $\begin{array}{l}\text { Spermatozoïdes } \\
\text { vivants (en \%) }\end{array}$ & $\begin{array}{l}\text { Concentration } \\
\left(\mathrm{x} 10^{6} \mathrm{spz}\right)\end{array}$ \\
\hline \multirow[t]{3}{*}{ F1 } & R1 & $3,13 \pm 0,50$ & $4,29 \pm 0,50$ & $90,81 \pm 3,22$ & $732,17 \pm 188,86$ \\
\hline & $\mathrm{R} 2$ & $3,05 \pm 0,46$ & $4,22 \pm 0,71$ & $91,15 \pm 3,63$ & $783,00 \pm 182,79$ \\
\hline & $\mathrm{R} 1-\mathrm{R} 2$ & $3,09 \pm 0,47$ & $4,25 \pm 0,61$ & $90,98 \pm 3,38$ & $757,58 \pm 184,82$ \\
\hline \multirow[t]{3}{*}{$\mathbf{F} 2$} & $\mathrm{R} 1$ & $3,17 \pm 0,77$ & $4,16 \pm 0,65$ & $91,17 \pm 2,41$ & $773,52 \pm 159,13$ \\
\hline & $\mathrm{R} 2$ & $3,33 \pm 0,58$ & $4,48 \pm 0,41$ & $88,96 \pm 4,28$ & $745,64 \pm 188,31$ \\
\hline & $\mathrm{R} 1-\mathrm{R} 2$ & $3,25 \pm 0,68$ & $4,32 \pm 0,56$ & $90,07 \pm 3,60$ & $759,58 \pm 172,25$ \\
\hline \multirow{3}{*}{$\mathbf{F 3}$} & $\mathrm{R} 1$ & $3,11 \pm 0,46$ & $4,27 \pm 0,41$ & $90,97 \pm 3,26$ & $781,23 \pm 139,97$ \\
\hline & R 2 & $3,19 \pm 0,63$ & $4,19 \pm 0,44$ & $90,04 \pm 3,42$ & $795,70 \pm 165,50$ \\
\hline & $\mathrm{R} 1-\mathrm{R} 2$ & $3,15 \pm 0,55$ & $4,23 \pm 0,42$ & $90,51 \pm 3,33$ & $788,47 \pm 151,11$ \\
\hline \multirow[t]{3}{*}{ F4 } & $\mathrm{R} 1$ & $3,05 \pm 0,43$ & $4,34 \pm 0,52$ & $90,39 \pm 3,31$ & $759,52 \pm 220,36$ \\
\hline & R 2 & $2,98 \pm 0,59$ & $4,19 \pm 0,42$ & $90,50 \pm 4,24$ & $753,64 \pm 217,90$ \\
\hline & $\mathrm{R} 1-\mathrm{R} 2$ & $3,01 \pm 0,51$ & $4,26 \pm 0,47$ & $90,44 \pm 3,75$ & $756,58 \pm 215,81$ \\
\hline \multirow[t]{3}{*}{ F5 } & $\mathrm{R} 1$ & $3,16 \pm, 50$ & $4,22 \pm 0,34$ & $87,41 \pm 5,02$ & $706,76 \pm 153,43$ \\
\hline & R 2 & $3,11 \pm 0,44$ & $4,31 \pm 0,42$ & $90,04 \pm 2,75$ & $777,58 \pm 178,88$ \\
\hline & $\mathrm{R} 1-\mathrm{R} 2$ & $3,14 \pm 0,46$ & $4,27 \pm 0,38$ & $88,72 \pm 4,20$ & $742,17 \pm 167,99$ \\
\hline \multirow[t]{3}{*}{ F6 } & $\mathrm{R} 1$ & $3,22 \pm 0,78$ & $4,16 \pm 0,55$ & $90,41 \pm 2,23$ & $790,29 \pm 199,22$ \\
\hline & R 2 & $3,09 \pm 0,59$ & $4,00 \pm 0,60$ & $89,57 \pm 4,39$ & $815,00 \pm 191,08$ \\
\hline & $\mathrm{R} 1-\mathrm{R} 2$ & $3,14 \pm 0,72$ & $4,08 \pm 0,59$ & $90,96 \pm 3,06$ & $870,41 \pm 246,62$ \\
\hline \multirow[t]{3}{*}{ F7 } & $\mathrm{R} 1$ & $3,15 \pm 0,50$ & $4,20 \pm 0,30$ & $87,60 \pm 5,00$ & $706,78 \pm 154,43$ \\
\hline & R 2 & $3,10 \pm 0,40$ & $4,30 \pm 0,40$ & $90,10 \pm 2,80$ & $777,60 \pm 175,80$ \\
\hline & $\mathrm{R} 1-\mathrm{R} 2$ & $3,15 \pm 0,35$ & $4,25 \pm 0,35$ & $88,75 \pm 4,25$ & $742,20 \pm 165,90$ \\
\hline \multirow[t]{3}{*}{ F8 } & $\mathrm{R} 1$ & $3,10 \pm 0,45$ & $4,30 \pm 0,50$ & $90,80 \pm 3,20$ & $732,14 \pm 188,80$ \\
\hline & R 2 & $3,20 \pm 0,63$ & $4,24 \pm 0,72$ & $91,10 \pm 3,60$ & $783,50 \pm 182,65$ \\
\hline & $\mathrm{R} 1-\mathrm{R} 2$ & $3,15 \pm 0,54$ & $4,25 \pm 0,60$ & $90,96 \pm 3,34$ & $777,60 \pm 184,82$ \\
\hline \multirow[t]{3}{*}{ F9 } & $\mathrm{R} 1$ & $3,15 \pm 0,76$ & $4,15 \pm 0,60$ & $91,18 \pm 2,40$ & $774,50 \pm 158,14$ \\
\hline & R 2 & $3,34 \pm 0,60$ & $4,45 \pm 0,42$ & $88,95 \pm 4,30$ & $745,60 \pm 187,30$ \\
\hline & $\mathrm{R} 1-\mathrm{R} 2$ & $3,24 \pm 0,70$ & $4,30 \pm 0,56$ & $90,08 \pm 3,65$ & $760,57 \pm 175,20$ \\
\hline \multirow[t]{3}{*}{ F10 } & $\mathrm{R} 1$ & $3,10 \pm 0,45$ & $4,28 \pm 0,43$ & $90,96 \pm 3,30$ & $780,30 \pm 140,90$ \\
\hline & R 2 & $3,20 \pm 0,62$ & $4,20 \pm 0,45$ & $90,02 \pm 3,40$ & $794,80 \pm 164,58$ \\
\hline & $\mathrm{R} 1-\mathrm{R} 2$ & $3,16 \pm 0,54$ & $4,25 \pm 0,40$ & $90,60 \pm 3,30$ & $790,40 \pm 150,10$ \\
\hline Moyenne F1-F10 & & $\mathbf{3 , 1 1} \pm \mathbf{0 , 5 7}$ & $\mathbf{4 , 3 0 \pm 0 , 5 1}$ & $90,20 \pm 3,62$ & $\mathbf{7 7 5 , 7 3} \pm \mathbf{1 8 8 , 0 4}$ \\
\hline
\end{tabular}


A. B. GBANGBOCHE et al. / Int. J. Biol. Chem. Sci. 5(5): 1871-1882, 2011

Tableau 3: Spermogramme comparé de certaines races bovines tropicales.

\begin{tabular}{|c|c|c|c|c|c|c|}
\hline Races & Volume (ml) & $\begin{array}{c}\text { Motilité de masse } \\
(05)\end{array}$ & $\begin{array}{c}\text { Concentration } \\
\left(\times 1^{6} \mathrm{spz}\right)\end{array}$ & $\begin{array}{c}\text { \% de spermatozoïdes } \\
\text { vivants }\end{array}$ & Pays ou région & Auteurs \\
\hline Baoulé & & 3,1 & 0,96 & 63,4 & Savane & Cloé et al., 1989 \\
\hline & 2,7 & & & & Burkina faso & \\
\hline Angoni & 3,25 & 2,9 & 1,7 & 81,5 & Nord Nigeria & $\begin{array}{l}\text { Igboeli et al., } \\
1971\end{array}$ \\
\hline Goudali & 6,5 & & 0,6 & 68,83 & Nord Nigeria & $\begin{array}{l}\text { KumiDiaka et al., } \\
1981\end{array}$ \\
\hline Créoles & 4,3 & 3 & 0,57 & 66 & Guadeloupe & $\begin{array}{l}\text { Gauthier et Varo, } \\
1985\end{array}$ \\
\hline cubaine & 5,88 & 4 & 0,81 & & Cuba & $\begin{array}{l}\text { MenendezBuxa- } \\
\text { dera et al., } 1983\end{array}$ \\
\hline Zébu Criollo & 5,85 & 4 & 0,90 & & Cuba & $\begin{array}{l}\text { MenendezBuxa- } \\
\text { dera et al., } 1983\end{array}$ \\
\hline $\begin{array}{l}\text { N'Dama x } \\
\text { N'Damance }\end{array}$ & 4,05 & 2,5 & 0,9 & 51,2 & Sudforestier & $\begin{array}{l}\text { Tamboura et al., } \\
1992\end{array}$ \\
\hline Muturu & $2,1 \pm 0,1$ & & $19,2 \pm 0,46$ & $34,5 \pm 1,6$ & Nord Nigeria & $\begin{array}{l}\text { Igboeli et al., } \\
1987\end{array}$ \\
\hline Borgou & $3,11 \pm 0,57$ & $4,24 \pm 0,51$ & $775,73 \pm 188,04$ & $90,18 \pm 3,61$ & Benin & $\begin{array}{l}\text { AdamouN'Diaye } \\
\text { et al., } 2000\end{array}$ \\
\hline
\end{tabular}




\section{DISCUSSION}

Caractéristiques des éjaculats et effet de la fréquence et du nombre de collecte

Les éjaculats obtenus sont conformes aux normes de Bishop (1961) et peuvent être utilisées pour l'insémination artificielle en semence fraîche dont les caractéristiques sont les suivantes: volume > à $1 \mathrm{ml}$; concentration > à $0,5 \times 10^{9}$ par ml ; motilité > à 3 ; pourcentage de spermatozoïdes vivants > à $70 \%$ (Jondet, 1980). Cette insémination artificielle en semence fraîche se fait notamment par voie cervicale puisque des volumes plus faibles de l'ordre de $0,5 \mathrm{ml}$ et $0,1 \mathrm{ml}$ de sperme introduits dans le cervix ont déjà donné depuis des décennies, d'aussi bons résultats que des volumes plus importants de l'ordre de 2 ou $4 \mathrm{ml}$ inséminés par voie vaginale (Jondet, 1980). Les taureaux Borgou ont présenté de meilleures concentrations et motilités (Tableau 3) que celles rapportées pour les taureaux N'Dama x N'Damance et Zébu Angoni (Tamboura et al., 1992). Outre l'effet du rang de collecte mis en exergue par Cloe et al. (1989) et qui n'est pas observé dans cette étude, d'autres facteurs susceptibles d'influencer la production spermatique sont évoqués en milieux tropicaux (Randel, 1990; Hanzen, 2009 ; 2010), comme le mode de collecte (Salisbury et Vandermark, 1961), le poids des animaux (Cloe et al., 1989), l'âge des animaux (Salisbury et Vandermark, 1961), l'hygrométrie (Cloe et al., 1989), la température (Igboeli et al., 1987 ; Coulibaly, 1988 ; Cloe et al., 1989), l'insolation et la pluviométrie (Coulibaly,1988), les saisons (Igboeli et al. 1987 ; Cloe et al., 1989), la température (Fagnemi et Adegbite, 1982; Menendez-Bruxadera et al., 1983 ; Tamboura et al., 1992), l'hygrométrie (Tamboura et al., 1982; Menendez-Bruxadera et al., 1983 ; Cloe et al., 1989) et la trypanosomose (Boly et al., 1991).

\section{Caractéristique des semences et effet des milieux de dilution}

Les valeurs de la longévité et la motilité des semences obtenues sont supérieures à celles communiquées sur des éjaculats en milieux tropicaux (Kumi-Diaka et al., 1981 ; Gauthier et Varo, 1985 ; Cloé et al., 1989; Tamboura et al., 1992). En technologie de perme, les dilueurs de par leur composition et leur nature (Quin et White, 1966) de même que le plasma séminal (Bishop 1961; Nunes, 1982) sont susceptibles d'avoir une action favorable ou défavorable sur les spermatozoïdes. La diminution de la motilité et de la viabilité des spermatozoïdes dans cette étude peut être la conséquence de l'altération de leur structure et leur état fonctionnel (Jondet, 1980), de la baisse de la viscosité des milieux de conservation, réduisant ainsi le métabolisme des spermatozoïdes, même si ces facteurs explicatifs n'ont pu être pris en considération dans notre étude. Pour les profils de la motilité (Figure 1) et de la viabilité (Figure 2) des spermatozoïdes dans les milieux à base de lait et de jaune d'œuf, l'effet semblable exercé par ces dilueurs est observé sur les spermatozoïdes $(\mathrm{p}>0,05)$, alors que Dauzier et al. (1954) ont signalé de meilleurs résultats avec la poudre de lait par rapport au jaune d'œuf pour une conservation de plusieurs heures à $+5^{\circ} \mathrm{C}$. Dans le cas de notre étude, la fraction lipidique et lipoprotéique du jaune d'œuf doit protéger les spermatozoïdes contre les chocs thermiques et va faciliter leur conservation (Moussa et al., 2002), c'est d'ailleurs pourquoi, l'ajout au dilueur 0,5 à $1 \%(\mathrm{~V} / \mathrm{V})$ de jaune d'œuf est suggéré afin de prolonger la survie des spermatozoïdes à la température ambiante et d'en apporter 5\% (V/V) pour la conservation à $+5{ }^{\circ} \mathrm{C}$ (Salisbury et al., 1961). Par contre chez les caprins l'enzyme EYC (Egg Yolk coaguling enzyme) retrouvée dans le plasma séminal des caprins peut hydrolyser la lécithine du jaune d'œuf en acide gras et lysolécithine avec un effet délétère sur la survie des spermatozoïdes (Jondet, 1980). Pour ce qui se rapporte au lait, il a l'avantage d'induire une capacitation précoce des spermatozoïdes (Burh et Zhao, 1992), parce qu'il contiend du 
citrate et une nouvelle addition du citrate de sodium n'est pas nécessaire (Jondet, 1980). Cependant, il peut contenir une faible teneur en glucose, élément énergétique métabolisable par les spermatozoïdes, et c'est pourquoi un ajout de glucose est recommandé (Palmer, 1984). En revanche, pour une conservation à $+5{ }^{\circ} \mathrm{C}$, l'addition d'autres sources d'énergie n'est pas reconnue nécessaire car Ehlers et Erb (1959) ont montré que la chute de la fertilité de 5,5\% pour les dilueurs lactés avec le fructose contre $4,1 \%$ pour les dilueurs sans fructose. Par ailleurs, en séparant par une dialyse les parties à faibles poids moléculaires du lait, son effet bénéfique à $+37{ }^{\circ} \mathrm{C}$ sur les spermatozoïdes du bélier et du taureau est signalé (Garcia et Graham, 1987) et des teneurs de $0,5 \%$ et $2 \%$ de caséine dans le lait sont optimales pour une conservation respective à $+37{ }^{\circ} \mathrm{C}$ et à $+5{ }^{\circ} \mathrm{C}$ (Martin, 1966). L'effet protecteur du lactosérum sur la semence est reconnu notamment pour le buffle (Bhosrekar et Ganguli, 1971) et pour conserver à $+5^{\circ} \mathrm{C}$, la fertilité des semences pendant plusieurs jours, l'addition de glycérol au dilueur lacté est recommandée (Jondet, 1980). La courte viabilité des spermatozoïdes dans le lait écrémé de commerce (Figure 2), peut s'expliquer par la destruction de la lactéine au cours de la pasteurisation, qui a la capacité de transformer le lactose en glucose et galactose, deux monosaccharides facilement métabolisables par les spermatozoïdes (Jondet, 1980). Par ailleurs, il est signalé chez les caprins, que l'une des protéines secrétées par les glandes bulbo-urétrale (protéine SBU III) n'est pas favorable à la survie in vitro des spermatozoïdes en présence de constituants lactés (Nunes, 1982), et reste à préciser chez les bovins. L'antifongique utilisé pour le lait de coco (Tableau1), n'a probablement pas stoppé, ni diminué la prolifération des champignons (Aspergillus flavus) retrouvés le plus souvent dans ce milieu et défavorise la survie des spermatozoïdes. Aussi, malgré la présence de protéines et des matières grasses en proportion variable dans le lait de coco (Fao, 2003), l'adjonction de $0,5 \%$ à $1,0 \%$ de jaune d'œuf dans le lait de coco pour une conservation à la température ambiante, et de $5 \%$ pour $+5 \quad{ }^{\circ} \mathrm{C}$ de conservation est recommandée (Norman et al., 1962), ce qui n'est pas observé dans notre étude. Même si les semences ne sont pas évaluées pour leur fécondance, d'autres auteurs ont obtenu après 5 jours de conservation à $+5{ }^{\circ} \mathrm{C}$ des spermatozoïdes dans le lait de coco, un taux de conception entre $55 \%$ et $72 \%$ au Kenya, Uganda, El Salvator, Paraguay, Inde et en Allemangne, de $67,7 \%$ pour les semences conservées pendant 4 jours entre $11^{\circ} \mathrm{C}$ et 25 ${ }^{\circ} \mathrm{C}$, et de $65,5 \%$ pour les semences congelées mises en paillettes (Salisbury et al., 1961). Par ailleurs, l'addition des substances oxydables comme le glucose, à la composition du lait de coco, a l'avantage de prolonger la viabilité des spermatozoïdes (Derivaux, 1971), surtout pour la semence bovine, en raison de sa haute concentration en spermatozoïdes, et un métabolisme anaérobie important où à $37{ }^{\circ} \mathrm{C}, \quad 10^{9}$ spermatozoïdes consomment $2 \mathrm{mg} / \mathrm{h}$ de fructose (Jondet, 1980). Pour les dilueurs à base de glucose à $5 \%$ et à $10 \%$, l'absence d'antibiotique peut favoriser la contamination bactérienne nuisible aux spermatozoïdes, toute chose qui reste à vérifier car l'adjonction d'antibiotique telles que la pénicilline et la streptomycine ou le mélange d'antibiotiques aux dilueurs a l'avantage d'inhiber le développement des germes banaux et spécifiques, puis de diminuer le pourcentage de retour en chaleur (Almquiste et al., 1951; Dunn et al., 1953). Le taux de jaune d'œuf à $15 \%$ (tableau 1) dans les dilueurs glucosés, peut être très faible et explique la faible viabilité et motilité des spermatozoïdes (Almquiste, 1962), d'où la nécessité de préciser la teneur en jaune d'œuf des dilueurs glucosés, car en milieux tempérés, la mobilité des spermatozoïdes 
d'étalon est passée de $8 \mathrm{~h}$ à $101 \mathrm{~h}$ dans une solution de glucose à $7 \%$, contre à $108 \mathrm{~h}$ dans le lait de jument, contre à $290 \mathrm{~h}$ dans une solution de glucose à $7 \%$ additionnée de jaune d'œuf à $5 \%$ et contre à $302 \mathrm{~h}$, dans le lait de jument additionné de jaune d'œuf à $5 \%$ (Kühr, 1957).

\section{Conclusion}

Les taureaux de race Borgou répondent à la cadence de collecte de sperme tous les 4 jours et ce qui correspond à 10 séances de récoltes en 36 jours. C'est un avantage dont le Centre d'Insémination Artificielle du Bénin peut tirer parti, pour majorer la production de semences des taureaux d'élite. Le citrate/fructose/jaune d'œuf, le lait de vache, le lait UHT commercial, le lait de coco, le glucose $5 \%$ et le glucose $10 \%$ qui sont les 6 milieux de dilution expérimentés peuvent être utilisés dans un délai de 48 heures de conservation à $+5^{\circ} \mathrm{C}$ et à 72 heures pour le lait de vache et le jaune d'œuf. Le lait de vache, facile à se procurer et peu cher, constitue un substitut éventuel pour le dilueur à base de citrate/fructose/jaune d'œuf, un produit dont la fourniture sur place n'est pas régulière. Les résultats in vitro devront être confrontés à des résultats de fertilité. L'équilibre entre la composition des dilueurs, la température de conservation et le conditionnement des semences ainsi diluées doit être élucidé de même que l'évolution du pH.

\section{REFERENCES}

Adamou-N'Diaye M, Gbangboche AB, Adjovi A, Hanzen CH. 2000. Caractéristiques spermatiques des taureaux de race Borgou au Bénin. Annales des Sciences Agronomiques du Bénin, 2 (1):71-83.

Adamou-N'diaye M, Gbangboche AB, Adjovi A, Jondet R. 2003. Crypréservation du sperme de taureau de race Borgou au Bénin. Revue Méd. Vét., 154 (1) : 3-8.
Almquist JO. 1951. A Comparison of penicillin, streptomycin and sulphanilamide for improving the fertility of semen from bulls of low fertility. J. Dairy Sci., 34: 819-822.

Almquist JO. 1962. Diluents for bovine semen. XI. Effects of glycerol on fertility and motility of spermatozoa in homogenized milk and skim milk. $J$. Dairy Sci., 45: 911-916.

Bishop DW. 1961. Biology of spermatozoa. In Sex and Internal Secretions (3e edn). The Williams and Wilkins Co. Edit.: USA; 722.

Boly H, Thiombiano D, Humblot P, Thibier M. 1991. Influence de Trypanosoma congolense sur la fonction sexuelle de taureaux Baoulé. Rev. Elev. Méd. Vét. Pays Trop., 44 (4): 475-480.

Bhosrekar M, Ganguli NC. 1971. Preservation of buffalo semen. Annuel repport, National Dairy Research Institute, Karnal 40-41, New-Delhi, Indian council of agriculture research.

Buhr MM, Zhao Y. 1992. Milk affect the calcium regulatory ability of bovine spermatozoa. La Haye. Abstract $\mathrm{n}^{\circ} 131$ : 417-419.

Cloe LC, Chicoteau P, Coulibaly M, Bassinga A. 1989. Caractéristiques spermatiques du taureau Baoulé (Bos taurus taurus) au Burkina Faso. Revue. Elev. Med. Vet. Pays Trop., 42 (3): 457462.

Coulibaly M. 1988 Recherche d'une base physiologique au saisonnement de la reproduction des Baoulé (Bos taurus). Mémoire de fin d'études, Institut du développement rural, Ouagadougou (Burkina-Faso), 79p.

Dauzier L, Thibault C, Winterberger S. 1954. Conservation du sperme de bélier après dilution et maintien de son pouvoir fécondant. Ann. Endocr. 15 : 341-350.

Derivaux J. 1971. Reproduction des Animaux Domestiques (Tomes 1 et 2). Edit. Derouaux : Liège. 
Dunn HO, Bratton RW, Henderson CR. 1953. Fertility of bovine spermatozoa in buffered whole egg extenders containing penicillin, streptomycin, sulphonamide and added glucose. J. Dairy Sci., 36: 524-530.

Ehlers MH, Erb RE. 1959. Influence of added fructose on fertility of bulls semen. A.I. Digest., 7(2): 9.

FAO. 2003. Cocos nucifera. http ://www.fao.ag/aga/agap/frg/afris/fr/D ata/498.htm, consulté le 12 octobre 2009.

Fayemi O, Adegbite O. 1982. Seasonal variations in sperm production of bulls in a tropical climate. Revue Elev. Méd. Vét. Pays Trop., 35 (1): 69 - 72.

Garcia MA, Graham EF. 1987. Effect of low molecular weight fractions from milk, egg yolk, and seminal plasma on freezability of bovine spermatozoa. Cryobiology, 24: 5, 429-436.

Gauthier D, Varo H. 1985. Caractéristiques spermatiques des taureaux en Guadeloupe. Variation avec la race. Ann. Zootech., 34 (4): 463 - 470.

Hanzen Ch. 2009. L'infertilité dans l'espèce bovine: un syndrome, 29p. Université de Liège, Faculté de médecine vétérinaire. http://www.therioruminant.ulg.ac.be/note s/200809/R16_Infertilite_bovine_2009.pd f. Consulté le 22 septembre 2011.

Hanzen Ch. 2010. Facteurs d'infertilité et d'infécondité en reproduction bovine. orbi.ulg.ac.be/bitstream/2268/70544/1/RO 8_Facteurs_generaux_2012.pdf. Consulté le 22 septembre 2011.

Igboeli G, Nwakalor LN, Orji BI, Onuora GI. 1987. Seasonal variation in the semen characteristics of Muturu (Bos brachyceros) bulls. Anim. Repr. Sci., 14: 31-38.

Iritani A, Nishikawa Y, Iritani, A, Nishikawa Y. 1961. Studies on the egg yolk coagulating factors in goat semen: II properties of the coagulating factor and influential conditions for coagulation. In Proceedings of Silver Jubilee Laboratory of Animal Husbandry, Kyoto University, pp.97-104.

Iritani A, Nishikawa Y. 1963. Studies on the egg-coagulating enzyme in goat semen; IV. On the position of yolk consitituents attacked by the coagulating enzyme. Jpn. J. Anim. Reprod. 8: 113-117.

Jondet J. 1980. Contribution à l'amélioration de la technologie du sperme de taureau. Thèse de Doctorat es Sciences, U. E.R. Sciences biologiques, Université de Rennes, France, 66 p.

Kühr J 1957. The characters of stallion semen and methods of diluting it. Anim. Breed. Abst., 25, 1692, 356.

Kumi-Diaka J, Nagaratnam V, Rwuaan JS. 1981. Seasonal and age related changes in semen quality and testicular morphology of bulls in a tropical environment. Vet. Rec., 108: 13 - 15.

Leboeuf B, Manfredi E, Boue P, Piacère A, Brice G, Baril G, Broqua C, Humblot P, Terqui M. 1998. L'insémination artificielle et l'amélioration génétique chez la chèvre laitière en France. INRA Prod. Anim., 11: 171-181.

Martin ICA. 1966. Diluents for the preservation of ram spermatozoa. I. Diluents used at $37{ }^{\circ} \mathrm{C}$ and $5{ }^{\circ} \mathrm{C}$ and containing casein. Aust. J. Biol. Sci., 19: 645-653.

Mc Fee AF, Swamson EW. 1960. The influence of cooling rates and storage temperatures on sperm activity. A. I. Digest, 8:12-14.

Menendez - Buxadera A, Morales JR, Perez AP, Guerra YD. 1983. Seasonal variation in semen production of Holstein, zebu and Criollo bulls under artificial insemination conditions in Cuba. Actes du colloque sur la Reproduction des ruminants en zone tropicale, Pointe - à Pitre, 8 - 10 juin 1983. Paris, INRA, Pp, 239 - 264. Les colloques de l'INRA $n^{\circ}$ 20.

Moussa M, Marinet V, Trimeche A, Tainturier D, Anton M. 2002. Low 
density lipoproteins extrated from egg yolk by easy method. Cryoprotective effect on frozen -thawed bull semen. Theriogenelogy, 57: 1695-1706.

Norman C, Johnson ID, Proterfield E, Goldberg RS, Dunbar Jr, Min HS. 1962. Survival and fertility of bovine sperm kept at variable temperatures in coconut milk extender. J. Agric. Sci., 59: 33-39.

Nunes J. 1982. Etude des effets du plasma séminal sur la survie in vitro des spermatozoïdes de bouc. Thèse de Doctorat, Université Pierre et Marie Curie, Paris, 33p.

Palmer 1984. Factors affecting stallion semen survival and fertility. In Proc. $10^{\text {th }}$ Congress on Anim. Repro and AI Urbana. Champaign, IL USA. (3) 377.

Quin PJ, White IG. 1966. The effect of cold shock and deep freezing on the concentration of major cations in spermatozoa. J. Reprod. Fert., 12 : 263270.

Salisbury GW, Vandemark NL. 1961. Physiology of Reproduction and Artificial Insemination of Cattle. Freeman \& Co: San Francisco (USA); $639 \mathrm{p}$.

SAS®. 2008. Statistical Analysis Systems Institute. User's guide, version 9.2 (TS2MO), Edition 2008. SAS®, Institute Inc., Cary, NC, USA.

Tamboura H, Zagret CT, Coulibaly M. 1992. Influence du climat tropical humide sur les caractéristiques spermatiques de races taurines en Côte-d'Ivoire. Bull. Anim. Hlth. Prod. Afr., 40 : 185 - 196. 